\title{
Environmental agents involved in the cause of primary biliary cirrhosis
}

\author{
Elias Kouroumalis \\ Professor of Gastroenterology, University of Crete Medical School, Head, Department Of Gastroenterology, \\ University Hospital, PO Box 1352, 71100 Heraklion, Crete, Greece \\ Fax: +30 2810 542085; E-mail: kouroum@meduocgr
}

\begin{abstract}
Primary biliary cirrhosis (PBC) is an immune mediated chronic cholestatic liver disease with a slowly progressive course It is a universal disease with a reported latitudinal gradient in prevalence and incidence.

The aetiology of primary biliary cirrhosis is still unknown. It is characterized by a $60 \%$ concordance in monozygotic twins and is considered an autoimmune disease because of several features common to other autoimmune conditions and the relatively homogeneous serological and biochemical features. However geoepidemiological and clinical studies strongly imply that environmental factors also play an important role. It is accepted that the disease is clearly the result of a combination of genetic and environmental factors.

Several risk factors have been suggested to be associated with $\mathrm{PBC}$, including exposure to infectious agents and chemical xenobiotics. This review will attempt to place such factors in perspective.
\end{abstract}

\section{Abbreviations}

$\begin{array}{ll}\text { AMA: } & \begin{array}{l}\text { Antimitochondrial antibodies } \\ \text { ExDK: }\end{array} \\ & \begin{array}{l}\text { Peptide sequence from PDC-E2 or } \\ \text { OGDC }\end{array} \\ \text { PDC-E2: } & \begin{array}{l}\text { E2 component of Pyruvate dehydro- } \\ \text { genase complex }\end{array} \\ \text { OGDC- } & \text { E2 component of 2-oxoglutarate de- } \\ \text { E2: } & \text { hydrogenase complex } \\ \text { MMTV: } & \text { mouse mammary tumour virus } \\ \text { UTIs: } & \text { Urinary tract infections } \\ \text { TLR: } & \text { Toll-like receptors }\end{array}$

\section{Introduction}

Primary biliary cirrhosis (PBC) is a chronic inflammatory autoimmune disease that mainly targets the cholangiocytes of the interlobular bile ducts in the liver [1]. Addison and Gull [2] have first described a disease with a PBC-like picture in 1851, but the term "primary biliary cirrhosis" was used in 1949 when a cohort of 18 patients with characteristic features of PBC was published [3].

Primary biliary cirrhosis, is considered to be a model autoimmune disease because of strong association with specific, marked female predominance, and frequent coexistence with other autoimmune conditions. Its pathogenesis remains largely unknown. It is assumed that PBC results from a multistep process, in which different combinations of genetic and environmental factors interact [4-6]. Genetic factors likely play an important role in conferring susceptibility to PBC, as indicated by the high concordance rate among monozygotic twins [7], the highest among autoimmune diseases and the increased incidence among first-degree relatives of affected individuals [8]. PBC often coexists with several other autoimmune diseases with a genetic background in the same individual or family [9]. However, the low concordance of PBC among discordant twin pairs [7], the geographical pattern in prevalence rate [10], and the occurrence of clusters of the disease [11] indicate that environmental factors might contribute to break immunological tolerance and lead to the onset of PBC. Clinical and experimental findings also support the implication of infectious agents or chemical compounds as possible triggering factors (6-9). A significant role for environmental factors was supported by the identification in the northeast of England of spatial nests of patients, using formal cluster analysis. The original UK analysis reported an in- 
creased frequency of PBC in former industrial and/or coal mining areas [12]. Space-time clustering also supports the importance of transient environmental factors in PBC. Space - time clustering is distinct from spatial clustering and is said to occur when an excess of cases is observed within small geographical areas over limited temporal periods, and this pattern cannot be explained by general excesses in those areas or at those periods [13].

Another recent study from New York examined the prevalence of PBC and PSC near superfund areas and reported significant clusters of $\mathrm{PBC}$ surrounding toxic sites [14]. In synopsis, these observations gave rise to the hypothesis of a chemical environmental factor, potentially associated with contaminated land, which could either trigger disease or cause disease through a direct toxic effect [15]. This hypothesis would also provide one possible explanation for the tissue tropism of PBC if the toxin or toxins are excreted into bile. Both chemicals [16-18] and bacteria [19] have been suggested as environmental factors responsible for the induction of PBC in genetically susceptible individuals.

However clinical findings should always be interpreted with caution since they usually represent associations that are very difficult to prove that are causally related to the disease. Moreover many associations are significant due to the large number of cases studied and many clinical parameters are reported in questionnaires and have not been verified by proper laboratory investigations.

The data currently available are based on the results of three main studies, two from the US [20,21] and one from the UK [22]. Other studies with smaller number of cases have also been reported. All of them have pointed out associations with some constitutive and environmental factors, among which family history of PBC, urinary tract infections [23,24] and tobacco [20] exposure were shown to be the most affecting. Beside these factors, a history of gravidity $[20,25]$, exogenous oestrogens [20,26], and frequent use of cosmetics [20, 21] have been also reported to be associated with an increased risk of the disease Environmental factors implicated in PBC are summarized in Table 1.

\section{Life style variables}

A strong relationship between $\mathrm{PBC}$ and smoking has been reported in studies from the USA and UK [21, 22]. Past smoking (OR 1569, 95\% CI 1292-1905) was weakly related to $\mathrm{PBC}$ in the American study [21].

\section{Table 1}

Environmental Factors implicated in the pathogenesis of Primary Biliary Cirrhosis

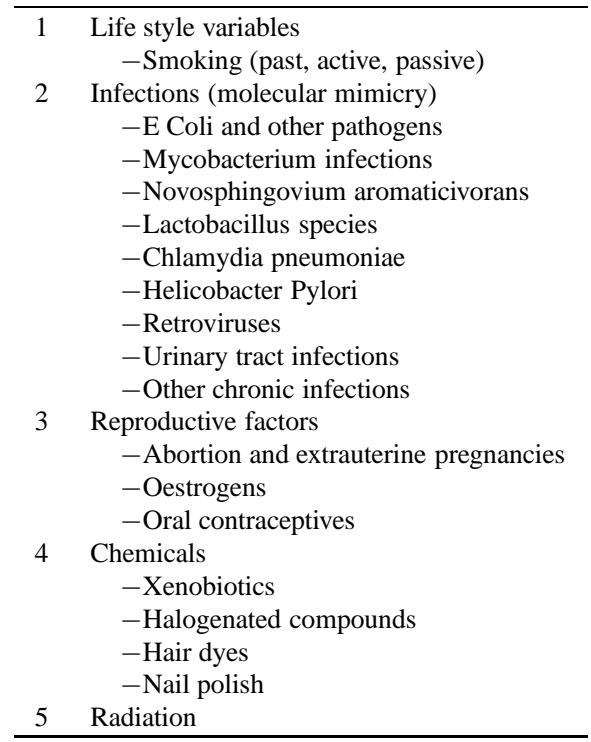

A recent case-control study of 222 patients and 509 matched controls [27] verified that individual smoking, either active or passive, was strongly associated with PBC (OR 31.95\% CI 20-50). Patients were more frequently active smokers or exposed daily to tobacco smoke. This association was independent of other associated variables, as age, gender, and alcohol consumption. The validation of this association in different populations strongly supports the hypothesis of a putative effect of some inhaled substances from tobacco on loss of immunological tolerance. Alternatively an association between PBC and smoking could also be explained by a possible effect of tobacco smoke on the T-helper-1 cytokine response that is predominant in PBC [28]. Further studies are required to determine whether smoking may worsen disease severity and progression as it has been recently suggested in a retrospective study [29].

\section{Infections}

The role of bacterial infections in PBC pathogenesis is supported by the significantly higher prevalence of urinary tract infections reported among patients and the presence of bacterial products in mononuclear cells surrounding damaged bile ducts [30].

Infectious agents, mainly bacteria, have been experimentally proved to induce autoimmune responses by a mechanism which is called molecular mimicry. Mi- 
crobes present peptides with different degrees of homology with self-proteins, leading to antibody production and cell-mediated immune response thus reacting with self-epitopes. T cell activation leads to crossreacting $\mathrm{T}$ cells and self-tissue destruction, thus perpetuating the autoimmune response [31]. The molecular mimicry hypothesis has been suggested based mostly on the experimental cross-reactivity of patient AMA and/or autoreactive $\mathrm{T}$ cells with prokaryotic antigens for several microbes, including E coli [32] which share an ExDK amino acid motif within their mimicry epitopes. This is the motif that is recognized by AMA and autoreactive T cells.

Of the bacterial strains suggested to lead to PBC through molecular mimicry, the most evidence has been reported for Escherichia coli, based on reports of an increased prevalence of urinary tract infections in patients with PBC [20]. Intestinal colonization by R(rough)forms E coli was found in the stool of all $22 \mathrm{PBC}$ patients vs 1 of 20 healthy controls and $25 \%$ of patients with other liver diseases [33]. Molecular mimicry and cross-reactivity of human PDC-E2 and E coli PDC-E2 were considered the main mechanisms in several studies. A low titer of AMA was measured in the sera of healthy women with a history of recurrent urinary tract infection regardless of any evidence for liver disease [24]. Affinity of anti-human PDC-E2 to E coli PDC-E2 was 100-fold higher in PBC patients [34]. T cell clones selected by human PDC-E2 were reactive with peptides of $\mathrm{E}$ coli PDC-E2 and E coli OGDCE2 [32], while T cell clones specific for E coli OGDCE2 were reactive with human mitochondrial autoantigens [35]. It should be noted however, that antibodies to E coli PDC are more frequent in the later stages of the disease and in low titers.

Besides E coli, a number of other bacteria have been shown as cross-reactive agents in PBC, including Proteus mirabilis, Klebsiella pneumoniae, Staphylococcus aureus, Neisseria meningitidis, Salmonella minnesota, Mycobacterium gordonae, and Trypanosoma brucei [19].

Mycobacterium infections, especially Mycobacterium gordonae, have been suggested to have a role in the pathogenesis of PBC. Cross-reactivity of sera from PBC patients with an extract of $\mathrm{M}$ gordonae, as well as cross-reactivity of antibodies to $\mathrm{M}$ gordonae heat-shock protein with human mitochondrial antibodies have been demonstrated [36]. Reactivity of sera from tuberculosis patients with the PDC-E2 subunit was reported by another group [37]. By contrast, others have failed to confirm serum reactivity of $\mathrm{PBC}$ patients with my- cobacteria, or to detect Mycobacterium DNA in the liver of PBC patients $[38,39]$.

Serological data suggest that a xenobiotic-metabolizing Gram-negative bacterium, Novosphingobium aromaticivorans, is so far the best candidate for the induction of PBC. It contains two proteins with the highest degree of homology with the major epitope of PDC-E2, and it can metabolize organic compounds and oestrogens thus possibly having a role in the well-documented female predominance of PBC. The reactivity against $\mathrm{N}$ aromaticivorans was 100- to 1000-fold higher than against E coli [40]. Serum reactivity against two lipoylated proteins of 47 and $50 \mathrm{KDa}$ from $\mathrm{N}$ aromaticivorans was observed in $100 \%$ of anti-PDC-E2 positive and in a fraction of AMA-negative sera, regardless of the disease stage [41]. It has also been reported that immunization with $\mathrm{N}$ aromaticivorans can induce autoreactive AMA and chronic PBC like T-cell-mediated autoimmunity against small bile ducts in a murine model of PBC [42].

Lactobacilli and Chlamydia, which show some structural homology with the autoantigen (although reactivity against them is considerably less than that against either $\mathrm{E}$ coli or $\mathrm{N}$ aromaticivorans), have also been implicated as putative pathogens, as has Helicobacter pylori [43-45].

Recently, a case of PBC following lactobacillus vaccination for recurrent vaginitis was reported The vaccine contained Lactobacillus salivarius, which exerts a high homology to the beta-galactosidase of Lactobacillus delbrueckii. Cross-reactivity of patients' autoantibodies against the human PDC-E2212-226 epitope and the beta-galactosidase of Lactobacillus delbrueckii was found. Affinity to the Lactobacillus epitope was higher than to the native mammalian, suggesting that antimicrobial reactivity may have preceded that to the selfmimic [46]. However, the AMA status of this patient before repetitive lactobacillus vaccination could not be assessed and causal relation of lactobacillus exposure and development of $\mathrm{PBC}$ remains speculative.

Chlamydia pneumoniae is a common cause of community-acquired pneumonia, which may have a role in chronic inflammatory processes such as atherosclerosis, multiple sclerosis and sarcoidosis. A potential role for $\mathrm{C}$ pneumoniae as a triggering or causative agent of PBC was suggested in one study by demonstrating CP-DNA in $100 \%$ of 39 liver biopsies of PBC patients, as compared to $85 \%$ of 105 controls biopsies [44]. By contrast, other studies failed to demonstrate correlation between $\mathrm{C}$ pneumoniae and PBC [47]. 
Helicobacter pylori infection is acquired in most cases during childhood and induces autoantibodies to the $\mathrm{H}+\mathrm{K}+\mathrm{ATPase}$ located in the gastric parietal cell. Microbial mimicry of $\mathrm{H}$ pylori peptides and human pyruvate dehydrogenase has been observed [48]. However, clinical or serological association could not be demonstrated, and seroprevalence of $\mathrm{H}$ pylori infection in 149 PBC patients was not increased compared to 619 healthy volunteers [49]. Two other studies failed to identify increased prevalence of $\mathrm{H}$ pylori DNA in liver tissues from PBC patients [38,50].

A novel human beta retrovirus was found in perihepatic lymph nodes and other biological samples from patients with PBC and has also been suggested in the aetiology of PBC [51] but this finding has been disputed by others [52]. Moreover, human beta-retrovirus has been recently found in the liver of patients affected by other liver diseases, including autoimmune hepatitis and viral hepatitis as well as healthy controls [30, 53], thus excluding a specific role of this virus in PBC. Also data of a randomised control trial of zidovudine and lamivudine in PBC patients do not convincingly support the role of a retrovirus in $\mathrm{PBC}$ pathogenesis [54]. Nevertheless, it is worth noting that the NODc3c4 mouse model of PBC [55] has been found to express. MMTV virus proteins in biliary epithelium [56]. These mice treated with anti MMTV had no evidence of the pathological traits of PBC, suggesting that MMTV triggers viral cholangitis in this experimental model [57]. MMTV replication is regulated in part by a progesterone-responsive glucocorticoid regulatory element in the promoter region, offering an alternative explanation for the female preponderance seen with PBC [58].

Several studies $[21,22,59]$, have indicated that urinary tract infections are associated with PBC. In the largest cohort of patients studied so far [21], a history of urinary tract infections was weakly associated with PBC (OR 1511,95\%CI 1192-1915). Vaginal infections were also more frequent in PBC patients. However it should be noted that, the history for urinary tract infections or vaginal infections reported by participants was not confirmed with laboratory data.

In the recent European case-control study, urinary tract infections were strongly associated with PBC (OR $27,95 \%$ CI 20-37). The proportion of individuals having experienced UTIs and the number of UTIs per person were significantly increased in patients compared to controls [27]. It may well be that urinary tract infectious agents, through a molecular mimicry mechanism $[31,60]$, may break immune tolerance and trigger autoimmunity in predisposed individuals [4].
It is still unknown whether other kinds of recurrent infection (nasopharyngitis for instance) could be associated with PBC development Compared to controls, patients with $\mathrm{PBC}$ reported significantly higher rates of tonsillectomy (57\% vs 50\%; $P=0018)$ and cholecystectomy ( $27 \%$ vs $17 \% ; P=0001)$ but these associations were not verified in the multivariate analysis [21]. Rates of tonsillectomy were reported similar in patients and controls in an other study [27] which however found significantly lower rate of reported vaccinations in the patients as compared to controls. This might be a further argument in favour of the involvement of a transient infectious agents in the pathogenesis of the disease.

Appendectomy, was significantly more frequently reported in patients with $\mathrm{PBC}$ in an epidemiological study in North America [20]. However, an earlier population-based case control study conducted in England did not show such associations [61]. More recently, another case - control study provided evidence that there was at least no association between PBC and the occurrence of appendectomy and pointed out the selection bias present in the previous study done in North America [62].

The involvement of innate immunity should also not be overlooked in the bacterial pathogenesis of PBC [63]. The innate immune system is able to detect pathogenic microbes and microbial molecules released by injured cells through toll-like and nod-like receptors The activation of toll-like receptor 9 in B cells stimulated with bacterial DNA $[64,65]$ results in an increased IgM production, a common laboratory finding in patients with PBC regardless of their AMA status. This effect is inhibited by the potassium channel blocker TRAM [65]. Further, monocytes from the peripheral blood of PBC patients secrete significantly higher levels of pro-inflammatory cytokines compared to controls when challenged with TLR ligands of both bacterial and viral origin [66], thus possibly facilitating the breakdown of tolerance in the presence of infectious agents.

\section{Reproductive factors}

A role for oestrogens in establishing the female predominance of PBC has been proposed earlier [26] but supporting evidence is limited. However conditions related to the female reproductive system have been associated with PBC. 
A history of abortion or extrauterine pregnancy has been also weakly associated with PBC(OR $20.95 \%$ CI 16-25), independently of smoking history [30]. This finding is consistent with an excess of menstrual abnormalities reported in women who will develop PBC [67]. This high rate of abortions or extrauterine pregnancy could be either a cause or a consequence of PBC. Never having been pregnant was significantly associated with protection from developing PBC (OR 0612, 95\% CI 0449-0834) [21].

PBC women seem to be younger than controls at their first pregnancy, as it has been reported in the US and Europe [10,21,27]. This intriguing finding has no obvious explanation It could suggest that exposure to fetal alloantigens is more likely to break immune tolerance in very young women, a hypothesis that might appear somewhat conflicting with the middle-age onset of the disease.

Use of oral oestrogens was significantly associated with an increased risk of PBC [24]. However this association was weak (OR 1548, 95\% CI 1273-1882) and may be due to the tendency of doctors to prescribe hormone replacement therapy against the possible bone loss of chronic cholestasis [68]. However, an altered distribution of oestrogen receptors has recently been demonstrated in liver samples of patients with different stages of PBC [69].

Interestingly in the more recent European study, use of oral contraceptives seemed to protect from PBC (OR 06, 95\% CI 05-08), and the difference was not related to smoking history. Because of the small number of cases and a relatively low level of statistical significance, cautious conclusions are required. Yet, this finding may suggest that, independently of their contraceptive properties, exogenous oestrogens may play a protective role against PBC development Such a hypothesis is consistent with the typical onset of the disease during menopause. It is furthermore supported by observations in humans and rodents suggesting that modulation of oestrogen receptors plays a critical role in cholangiocyte proliferation and that ductopenia might be favored by oestrogenic deficiency $[69,70]$.

\section{Chemicals}

Among non genetic factors, several lines of evidence spanning from geoepidemiology to experimental findings support the role of xenobiotics, ie, chemicals that are capable to induce molecular mimicry through cross reactivity [71].
Xenobiotics are foreign compounds that may alter or complex with self or non-self-proteins. The result is an alteration in the molecular structure of the native protein capable to induce neo antigens and elicit an immune response leading to the cross-recognition of the self form, which could in turn perpetuate the immune response [18].

Although it is not clear how xenobiotics or the modified cellular proteins initiate autoimmunity in PBC, several mechanisms have been proposed. Firstly, the direct toxic effect of the xenobiotics or the neo-antigens my cause abnormal cell death by apoptosis or necrosis, conditions favouring generation of immunogenic auto-epitopes. Secondly, the neo antigen- specific $\mathrm{T}$ cells and B cells, once primed, may cross react with the less immunogenic native autoantigen. Lastly, chemical modification of the native cellular protein such as removal and/or exchange of a hapten on the native peptide have been shown to change processing in antigen presenting cells and lead to the presentation of cryptic peptides [72].

In accordance with the above a specific halogenated organic compound was shown to elicit AMA production from patients with $\mathrm{PBC}$ once attached to the major mitochondrial epitope. This neoantigen was experimentally shown to be recognized by sera from PBC patients with a higher affinity than native forms of such antigens [16]. Moreover one such halogenated compound was capable of inducing AMA production in animal models $[17,73,74]$ without requiring the peptide backbone of PDC-E2, but failed to produce liver lesions and disappeared when the stimulus was discontinued [75]. An elegant study was able to demonstrate that recombinant lipoylation enzymes can effectively replace xenobiotics in lieu of lipoic acid in the PDC-E2 complex and thus provide a crucial proof of concept for xenobiotic initiation of PBC [76]. A different approach reported the induction of PBC-like liver lesions following longer follow-ups in guinea pigs [77], whereas a further study reported two new xenobiotic-induced PBC murine models based on the immunization with 2octynoic acid of NOD1101 or C57BL/6 mice [78,79]. These two most recent models share the breakdown of tolerance in the absence of PDC-E2 molecules but fail to manifest the progression to liver cirrhosis.

2-nonynoic acid was also shown to be capable of being recognized by PBC sera with high affinity [80, 81]. This is particularly interesting because this nonnaturally occurring compound is known to be found in several cosmetic products (including certain nail polish products) 
Use of hair dyes has also been associated with PBC [22] but this association was very weak and not supported by multivariate analysis in the large American study [21] while it was disputed in the recent large European study [27].

The frequent use of nail polish slightly increased the risk of having PBC [21]. However, the odds ratio for increased frequency of nail polish use was low and this association must be interpreted with caution Nonetheless, these data are intriguing in view of the xenobiotics hypothesis proposed for the development PBC with specific halogenated compounds that could increase the immunogenicity of mitochondrial proteins and able to induce AMA in animal models [16,73].

Radiation may also be a risk factor for PBC. In a study from Japan the prevalence of PBC was higher among atomic bomb survivors in Nagasaki compared to the general population [1]. Verification of these findings is difficult to be obtained. Only a repetition of this study in areas of nuclear accidents could offer further evidence.

\section{Cancers}

An initial report of an association of $\mathrm{PBC}$ with breast cancer [82] was not confirmed by others $[21,27,83]$.

\section{Conclusions}

The autoimmune pathogenesis is supported by a plethora of experimental and clinical data, such as the presence of autoreactive $\mathrm{T}$ cells and serum autoantibodies. The aetiology remains unknown, although evidence suggests a role for both genetic susceptibility and environmental factors that remain to be determined. In fact, a number of chemicals and infectious agents have been proposed to induce the disease in predisposed individuals.

The role of identified environmental risk factors should be investigated in animal studies to achieve a satisfactory model for the etiology of PBC. Investigations of putative environmental agents in different genetically modified mouse strains could uncover the geneticenvironmental interactions leading to the development of PBC.

\section{References}

[1] K. Ohba, K. Omagari, H. Kinoshita et al., Primary biliary cirrhosis among atomic bomb survivors in nagasaki, japan, Journal of Clinical Epidemiology 54 (2001), 845-850.

[2] T. Addison and W. Gull, On a certain affection of the skinvitiligoidea- $\alpha$-plana $\beta$-tuberosa, Guys Hosp Rep 7 (1851), G265-G277.

[3] E.H. Ahrens, Jr. and H.G. Kunkel, The relationship between serum lipids and skin xanthomata in 18 patients with primary biliary cirrhosis, The Journal of Clinical Investigation $\mathbf{2 8}$ (1949), 1565-1574.

[4] M.E. Gershwin and I.R. Mackay, The causes of primary biliary cirrhosis: Convenient and inconvenient truths, Hepatology (Baltimore, Md 47 (2008), 737-745.

[5] S. Hohenester, R.P. Oude-Elferink and U Beuers, Primary biliary cirrhosis, Seminars in Immunopathology 31 (2009), 283-307.

[6] T. Kumagi and E.J. Heathcote, Primary biliary cirrhosis, Orphanet Journal of rare Diseases 3 (2008), 1.

[7] C. Selmi, M.J. Mayo, N. Bach et al., Primary biliary cirrhosis in monozygotic and dizygotic twins: Genetics, epigenetics, and environment, Gastroenterology 127 (2004), 485-492.

[8] P. Invernizzi, C. Selmi, I.R. Mackay, M. Podda and M.E. Gershwin, From bases to basis: Linking genetics to causation in primary biliary cirrhosis, Clin Gastroenterol Hepatol 3 (2005), 401-410.

[9] J.A. Talwalkar and K.D. Lindor, Primary biliary cirrhosis, Lancet 362 (2003), 53-61.

[10] C. Selmi, P. Invernizzi, E.B. Keeffe et al., Epidemiology and pathogenesis of primary biliary cirrhosis, Journal of Clinical Gastroenterology 38 (2004), 264-271.

[11] S. Abu-Mouch, C. Selmi, G.D. Benson et al., Geographic clusters of primary biliary cirrhosis, Clinical \& Developmental Immunology 10 (2003), 127-131.

[12] M.I. Prince, A. Chetwynd, P. Diggle, M. Jarner, J.V. Metcalf and O.F. James, The geographical distribution of primary biliary cirrhosis in a well-defined cohort, Hepatology (Baltimore, Md 34 (2001), 1083-1088.

[13] R.J. McNally, S. Ducker and O.F. James, Are transient environmental agents involved in the cause of primary biliary cirrhosis? Evidence from space-time clustering analysis, Hepatology (Baltimore, Md 50 (2009), 1169-1174.

[14] A. Ala, C.M. Stanca, M. Bu-Ghanim et al, Increased prevalence of primary biliary cirrhosis near superfund toxic waste sites, Hepatology (Baltimore, Md 43 (2006), 525-531.

[15] D.E Jones, Pathogenesis of primary biliary cirrhosis, Gut 56 (2007), 1615-1624.

[16] A. Long, C. Quan, J. Van de Water et al., Immunoreactivity of organic mimeotopes of the e 2 component of pyruvate dehydrogenase: Connecting xenobiotics with primary biliary cirrhosis, J Immunol 167 (2001), 2956-2963.

[17] S.A. Long, J. Van de Water and M.E. Gershwin, Antimitochondrial antibodies in primary biliary cirrhosis: The role of xenobiotics, Autoimmunity Reviews 1 (2002), 37-42.

[18] R. Rieger and M.E. Gershwin, The $x$ and why of xenobiotics in primary biliary cirrhosis, Journal of Autoimmunity 28 (2007), 76-84.

[19] C. Selmi and M.E. Gershwin, Bacteria and human autoimmunity: The case of primary biliary cirrhosis, Current Opinion in Rheumatology 16 (2004), 406-410.

[20] A. Parikh-Patel, E.B. Gold, H. Worman, K.E. Krivy and M.E. Gershwin, Risk factors for primary biliary cirrhosis in a cohort 
of patients from the united states, Hepatology (Baltimore, $M d$ 33 (2001), 16-21.

[21] M.E. Gershwin, C. Selmi, H.J. Worman et al, Risk factors and comorbidities in primary biliary cirrhosis: A controlled interview-based study of 1032 patients, Hepatology (Baltimore, $M d 42$ (2005), 1194-1202.

[22] O. James, S. Ducker and M. Prince, Case control studies support the association of environmental and genetic risk factors with primary biliary cirrhosis, Hepatology (Baltimore, $M d \mathbf{4 2}$ (Suppl 1) (2005), 209A.

[23] N. Agmon-Levin, B.S. Katz and Y. Shoenfeld, Infection and primary biliary cirrhosis, Isr Med Assoc J 11 (2009), 112-115.

[24] P. Butler, F. Valle, J.M. Hamilton-Miller, W. Brumfitt, H. Baum and A.K. Burroughs, M2 mitochondrial antibodies and urinary rough mutant bacteria in patients with primary biliary cirrhosis and in patients with recurrent bacteriuria, Journal of Hepatology 17 (1993), 408-414.

[25] A. Parikh-Patel, E. Gold, J. Utts and M.E. Gershwin, The association between gravidity and primary biliary cirrhosis, Annals of Epidemiology 12 (2002), 264-272.

[26] J.M. Guattery and W.W. Faloon, Effect of estradiol upon serum enzymes in primary biliary cirrhosis, Hepatology (Baltimore, Md 7 (1987), 737-742.

[27] C. Corpechot, Y. Chretien, O. Chazouilleres and R. Poupon, Demographic, lifestyle, medical and familial factors associated with prim ary biliary cirrhosis, Journal of hepatology 53, 162-169.

[28] K. Harada, J. Van de Water, P.S. Leung et al., In situ nucleic acid hybridization of cytokines in primary biliary cirrhosis: Predominance of the th1 subset, Hepatology (Baltimore, $M d$ 25 (1997), 791-796.

[29] C.O. Zein, K. Beatty, A.B. Post, L. Logan, S. Debanne and A.J. McCullough, Smoking and increased severity of hepatic fibrosis in primary biliary cirrhosis: A cross validated retrospective assessment, Hepatology (Baltimore, Md 44 (2006), 1564-1571.

[30] J. McDermid, M. Chen, Y. Li et al., Reverse transcriptase activity in patients with primary biliary cirrhosis and other autoimmune liver disorders, Alimentary Pharmacology \& Therapeutics 26 (2007), 587-595.

[31] J. Van de Water, H. Ishibashi, R.L. Coppel and M.E. Gershwin, Molecular mimicry and primary biliary cirrhosis: Premises not promises, Hepatology (Baltimore, $M d \mathbf{3 3}$ (2001), 771-775.

[32] S. Shimoda, M. Nakamura, H. Shigematsu et al., Mimicry peptides of human pdc-e2 163-176 peptide, the immunodominant t-cell epitope of primary biliary cirrhosis, Hepatology (Baltimore, Md 31 (2000), 1212-1216.

[33] U. Hopf, B. Moller, R. Stemerowicz et al., Relation between escherichia coli r(rough)-forms in gut, lipid a in liver, and primary biliary cirrhosis, Lancet 2 (1989), 1419-1422.

[34] H. Miyakawa, A. Tanaka, C. Selmi et al., Serum reactivity against bacterial pyruvate dehydrogenase: Increasing the specificity of anti-mitochondrial antibodies for the diagnosis of primary biliary cirrhosis, Clinical \& Developmental Immunology 13 (2006), 289-294

[35] H. Tanimoto, S. Shimoda, M. Nakamura et al., Promiscuous t cells selected by escherichia coli: Ogdc-e2 in primary biliary cirrhosis, Journal of Autoimmunity 20 (2003), 255-263.

[36] L. Vilagut, A. Pares, O. Vinas, J. Vila, M.T. Jimenez de Anta and J. Rodes, Antibodies to mycobacterial 65-kd heat shock protein cross-react with the main mitochondrial antigens in patients with primary biliary cirrhosis, European Journal of Clinical Investigation 27 (1997), 667-672.
[37] R. Klein, M. Wiebel, S. Engelhart and P.A. Berg, Sera from patients with tuberculosis recognize the m2a-epitope (e2-subunit of pyruvate dehydrogenase) specific for primary biliary cirrhosis, Clinical and Experimental Immunology 92 (1993), 308316.

[38] A. Tanaka, T.P. Prindiville, R. Gish et al., Are infectious agents involved in primary biliary cirrhosis? A pcr approach, Journal of Hepatology 31 (1999), 664-671.

[39] J. O'Donohue, B. McFarlane, A. Bomford, M. Yates and R. Williams, Antibodies to atypical mycobacteria in primary biliary cirrhosis, Journal of Hepatology 21 (1994), 887-889.

[40] C. Selmi, D.L. Balkwill, P. Invernizzi et al., Patients with primary biliary cirrhosis react against a ubiquitous xenobioticmetabolizing bacterium, Hepatology (Baltimore, Md 38 (2003), 1250-1257.

[41] K.A. Padgett, C. Selmi, T.P. Kenny et al., Phylogenetic and immunological definition of four lipoylated proteins from novosphingobium aromaticivorans, implications for primary biliary cirrhosis, Journal of Autoimmunity 24 (2005), 209219.

[42] J. Mattner, P.B. Savage, P. Leung et al., Liver autoimmunity triggered by microbial activation of natural killer $\mathrm{t}$ cells, Cell host \& Microbe 3 (2008), 304-315.

[43] P.S. Leung, O. Park, S. Matsumura, A.A. Ansari, R.L. Coppel and M.E. Gershwin, Is there a relation between chlamydia infection and primary biliary cirrhosis?, Clinical \& Developmental Immunology 10 (2003), 227-233.

[44] A.S. Abdulkarim, L.M. Petrovic, W.R. Kim, P. Angulo, R.V. Lloyd and K.D. Lindor, Primary biliary cirrhosis: An infectious disease caused by chlamydia pneumoniae?, Journal of Hepatology 40 (2004), 380-384.

[45] K. Dohmen, H. Shigematsu, Y. Miyamoto, F. Yamasaki, K. Irie and H. Ishibashi, Atrophic corpus gastritis and helicobacter pylori infection in primary biliary cirrhosis, Digestive Diseases and Sciences 47 (2002), 162-169.

[46] D. Bogdanos, T. Pusl, C. Rust, D. Vergani and U. Beuers, Primary biliary cirrhosis following lactobacillus vaccination for recurrent vaginitis, Journal of Hepatology 49 (2008), 466473.

[47] D. Taylor-Robinson, A.W. Sharif, N.S. Dhanjal and S.D. Taylor-Robinson, Chlamydia pneumoniae infection is an unlikely cause of primary biliary cirrhosis, Journal of Hepatology 42 (2005), 779-780.

[48] D.P. Bogdanos, H. Baum, A. Grasso et al., Microbial mimics are major targets of crossreactivity with human pyruvate dehydrogenase in primary biliary cirrhosis, Journal of Hepatology 40 (2004), 31-39.

[49] M. Durazzo, F. Rosina, A. Premoli et al., Lack of association between seroprevalence of helicobacter pylori infection and primary biliary cirrhosis, World J Gastroenterol 10 (2004), 3179-3181.

[50] S.Y. Boomkens, S. de Rave, R.G. Pot et al., The role of helicobacter spp In the pathogenesis of primary biliary cirrhosis and primary sclerosing cholangitis, FEMS Immunology and Medical Microbiology 44 (2005), 221-225.

[51] L. Xu, Z. Shen, L. Guo et al., Does a betaretrovirus infection trigger primary biliary cirrhosis? Proceedings of The National Academy of Sciences of The United States of America $\mathbf{1 0 0}$ (2003), 8454-8459.

[52] C. Selmi, S.R. Ross, A.A. Ansari et al., Lack of immunological or molecular evidence for a role of mouse mammary tumor retrovirus in primary biliary cirrhosis, Gastroenterology 127 (2004), 493-501. 
[53] H. Johal, G.M. Scott, R. Jones, C. Camaris, S. Riordan and W.D.. Rawlinson, Mouse mammary tumour virus-like virus (mmtv-lv) is present within the liver in a wide range of hepatic disorders and unrelated to nuclear p53 expression or hepatocarcinogenesis, Journal of Hepatology 50 (2009), 548-554.

[54] A.L. Mason, K.D. Lindor, B.R. Bacon, C. Vincent, J.M. Neuberger and S.T. Wasilenko, Clinical trial: Randomized controlled trial of zidovudine and lamivudine for patients with primary biliary cirrhosis stabilized on ursodiol, Alimentary Pharmacology \& Therapeutics (2008).

[55] J. Irie, Y. Wu, L.S. Wicker et al., NodC3c4 congenic mice develop autoimmune biliary disease that serologically and pathogenetically models human primary biliary cirrhosis, The Journal of Experimental Medicine 203 (2006), 1209-1219.

[56] M. Chen, D. Graham, G. Zhang, S. Wasilenko, C. McDougall and M. Kneteman, Biliary infection with mouse mammary tumor virus in the nodC $3 \mathrm{c} 4$ mouse and other mouse models of primary biliary cirrhosis, Hepatology (Baltimore, Md $\mathbf{4 6}$ (2007), 551A.

[57] B. Subsin, M. Chen, S. Girgis and A. Mason, Mouse mammary tumor virus triggers viral cholangitis in the nodC $3 \mathrm{c} 4$ mouse model of primary biliary cirrhosis, Hepatology (Baltimore, Md 50 (2009), 998A.

[58] C. Scheidereit, D. von der Ahe, A.C. Cato et al., Protein-DNA interactions at steroid hormone regulated genes, Endocrine Research 15 (1989), 417-440.

[59] A.K. Burroughs, I.J. Rosenstein, O. Epstein, J.M. HamiltonMiller, W. Brumfitt and S. Sherlock, Bacteriuria and primary biliary cirrhosis, Gut 25 (1984), 133-137.

[60] J. Van de Water, J. Turchany, P.S. Leung et al., Molecular mimicry in primary biliary cirrhosis Evidence for biliary epithelial expression of a molecule cross-reactive with pyruvate dehydrogenase complex-e2, The Journal of Clinical Investigation 91 (1993), 2653-2664.

[61] D. Howel, C.M. Fischbacher, R.S. Bhopal, J. Gray, J.V. Metcalf and O.F. James, An exploratory population-based casecontrol study of primary biliary cirrhosis, Hepatology (Baltimore, Md 31 (2000), 1055-1060.

[62] E.I. Rigopoulou, S.P. Georgiadou, S. Barbanis and G.N. Dalekos, Lack of association between appendectomy and primary biliary cirrhosis, Scandinavian Journal of Gastroenterology 41 (2006), 573-576.

[63] M. Ehlers and J.V. Ravetch, Opposing effects of toll-like receptor stimulation induce autoimmunity or tolerance, Trends in Immunology 28 (2007), 74-79.

[64] S. Agrawal and E.R. Kandimalla, Modulation of toll-like receptor 9 responses through synthetic immunostimulatory motifs of DNA, Annals of The New York Academy of Sciences 1002 (2003), 30-42.

[65] Y. Moritoki, Z.X. Lian, H. Wulff et al., AMA production in primary biliary cirrhosis is promoted by the TLR9 ligand $\mathrm{CpG}$ and suppressed by potassium channel blockers, Hepatology (Baltimore, Md 45 (2007), 314-322.

[66] T.K. Mao, Z.X. Lian, C. Selmi et al., Altered monocyte responses to defined TLR ligands in patients with primary biliary cirrhosis, Hepatology (Baltimore, Md 42 (2005), 802-808.

[67] A.J. Stellon and R. Williams, Increased incidence of menstrual abnormalities and hysterectomy preceding primary biliary cirrhosis, British Medical Journal (Clinical Research Ed 293 (1986), 297-298.
[68] K.V. Menon, P. Angulo, G.M. Boe and K.D. Lindor, Safety and efficacy of estrogen therapy in preventing bone loss in primary biliary cirrhosis, The American Journal of gastroenterology 98 (2003), 889-892.

[69] D. Alvaro, P. Invernizzi, P. Onori et al., Estrogen receptors in cholangiocytes and the progression of primary biliary cirrhosis, Journal of Hepatology 41 (2004), 905-912.

[70] D. Alvaro, P. Onori, V.D. Metalli et al., Intracellular pathways mediating estrogen-induced cholangiocyte proliferation in the rat, Hepatology (Baltimore, Md 36 (2002), 297-304.

[71] C. Selmi, C.A. Cocchi, M. Zuin and M.E. Gershwin, The chemical pathway to primary biliary cirrhosis, Clinical Reviews in Allergy \& Immunology 36 (2009), 23-29.

[72] P. Griem, M. Wulferink, B. Sachs, J.B. Gonzalez and E. Gleichmann, Allergic and autoimmune reactions to xenobiotics: How do they arise? Immunology Today 19 (1998), 133-141.

[73] P.S. Leung, C. Quan, O. Park et al., Immunization with a xenobiotic 6-bromohexanoate bovine serum albumin conjugate induces antimitochondrial antibodies, J Immunol 170 (2003), 5326-5332.

[74] P. Invernizzi, C. Selmi and M.E. Gershwin, Update on primary biliary cirrhosis, Dig Liver Dis 42, 401-408.

[75] K. Amano, P.S. Leung, Q. Xu et al., Xenobiotic-induced loss of tolerance in rabbits to the mitochondrial autoantigen of primary biliary cirrhosis is reversible, J Immunol 172 (2004), 6444-6452.

[76] H.R. Walden, J.A. Kirby, S.J. Yeaman, J. Gray, D.E. Jones and J.M. Palmer, Xenobiotic incorporation into pyruvate dehydrogenase complex can occur via the exogenous lipoylation pathway, Hepatology (Baltimore, Md 48 (2008), 1874-1884.

[77] P.S. Leung, O. Park, K. Tsuneyama et al., Induction of primary biliary cirrhosis in guinea pigs following chemical xenobiotic immunization, J Immunol 179 (2007), 2651-2657.

[78] K. Wakabayashi, Z.X. Lian, P.S. Leung et al., Loss of tolerance in $c 57 \mathrm{bl} / 6$ mice to the autoantigen 22 subunit of pyruvate dehydrogenase by a xenobiotic with ensuing biliary ductular disease, Hepatology (Baltimore, Md 48 (2008), 531-540.

[79] K. Wakabayashi, K. Yoshida, P.S. Leung et al., Induction of autoimmune cholangitis in non-obese diabetic (nod)1101 mice following a chemical xenobiotic immunization, Clinical and Experimental Immunology 155 (2009), 577-586.

[80] K. Amano, P.S. Leung, R. Rieger et al., Chemical xenobiotics and mitochondrial autoantigens in primary biliary cirrhosis: Identification of antibodies against a common environmental, cosmetic, and food additive, 2-octynoic acid, J Immunol 174 (2005), 5874-5883.

[81] R. Rieger, P.S. Leung, M.R. Jeddeloh et al., Identification of 2nonynoic acid, a cosmetic component, as a potential trigger of primary biliary cirrhosis, Journal of Autoimmunity 27 (2006), 7-16.

[82] P.R. Mills, P. Boyle, E.M. Quigley et al., Primary biliary cirrhosis: An increased incidence of extrahepatic malignancies?, Journal of clinical pathology 35 (1982), 541-543.

[83] A. Floreani, M.R. Biagini, M. Chiaramonte, S. Milani, C. Surrenti and R. Naccarato, Incidence of hepatic and extrahepatic malignancies in primary biliary cirrhosis (PBC), The Italian Journal of Gastroenterology 25 (1993), 473-476. 


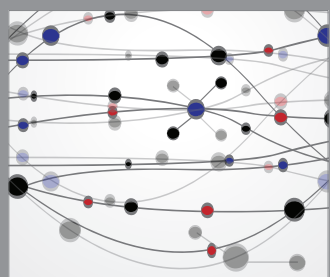

The Scientific World Journal
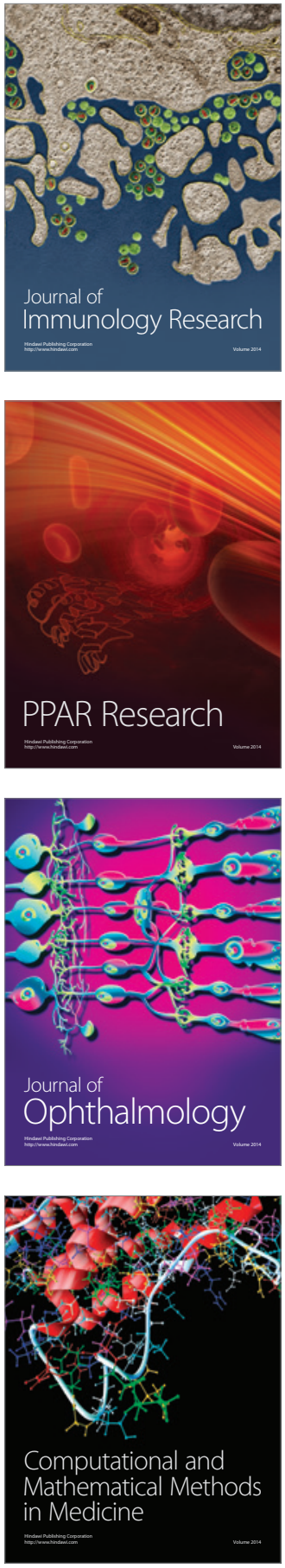

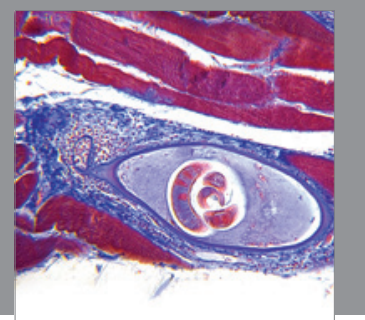

Gastroenterology

Research and Practice
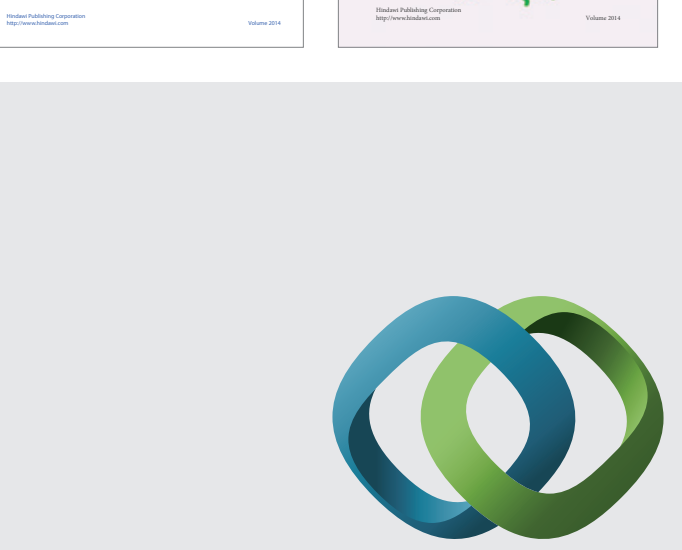

\section{Hindawi}

Submit your manuscripts at

http://www.hindawi.com
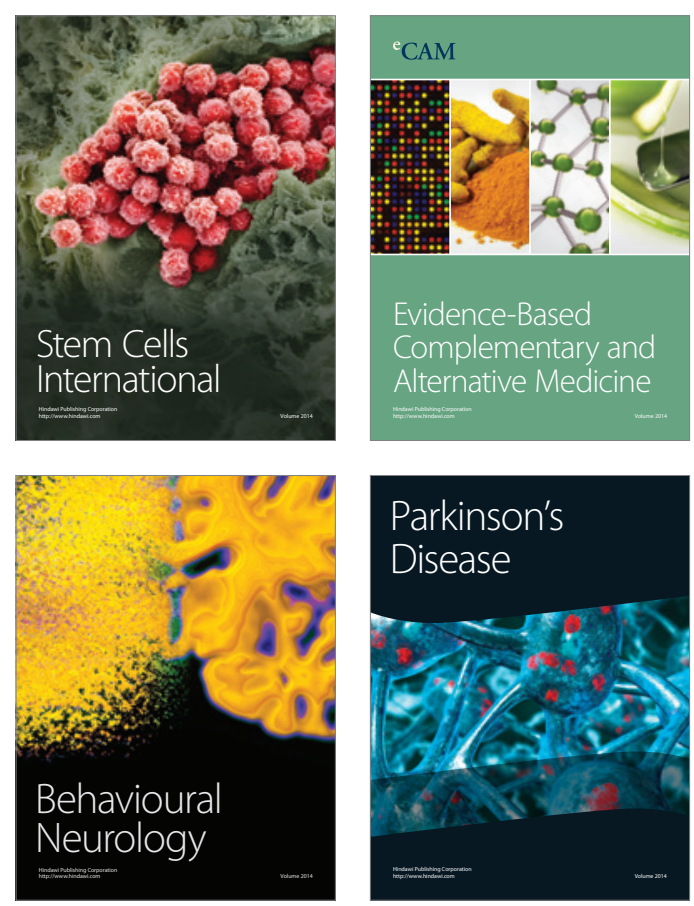

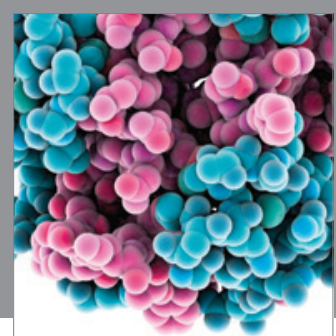

Journal of
Diabetes Research

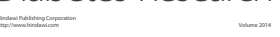

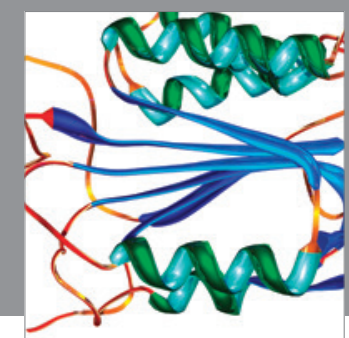

Disease Markers
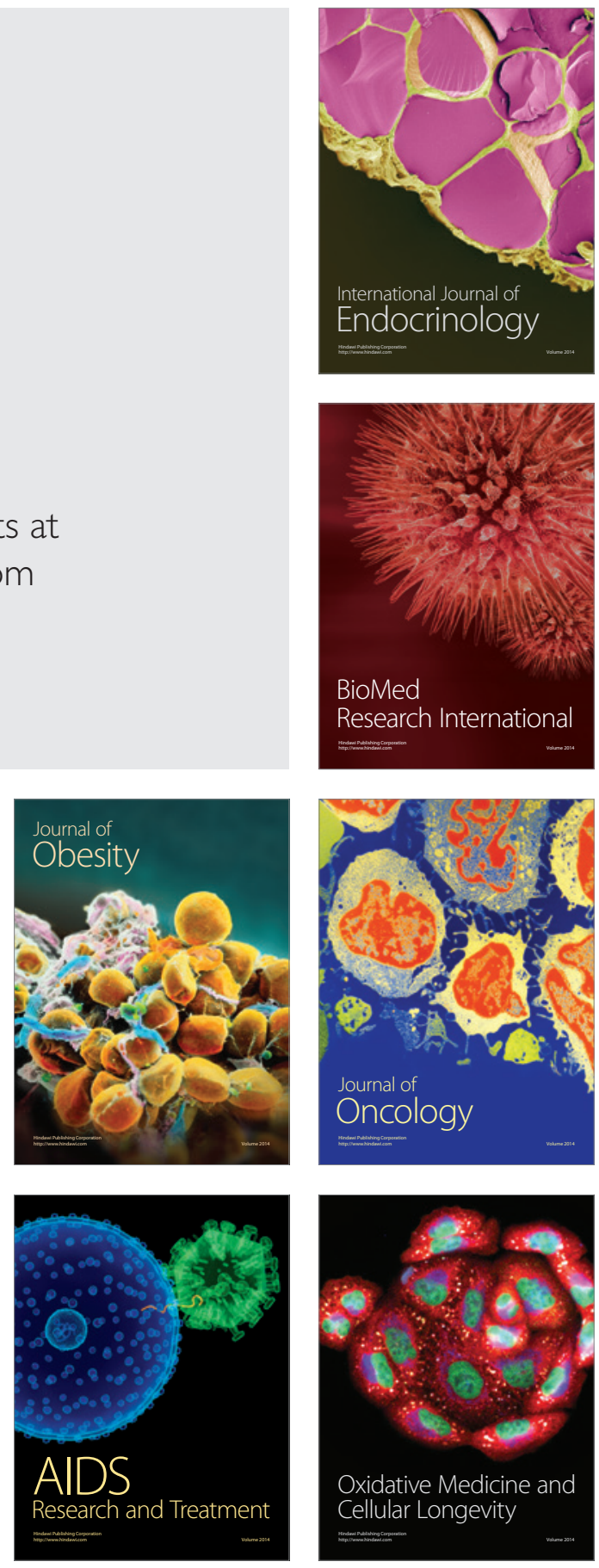